\title{
TIME DELAY OF PHOTONS OF DIFFERENT ENERGIES IN MULTIDIMENSIONAL COSMOLOGICAL MODELS
}

\author{
T. Harko and K. S. Cheng \\ Department of Physics, University of Hong Kong, Pokfulam Road, Hong Kong, China; harko@hkucc.hku.hk, hrspksc@hkucc.hku.hk \\ Received 2003 December 1; accepted 2004 May 4
}

\begin{abstract}
We consider the general expressions for the time delay of photons of different energies in the framework of multidimensional cosmological models. In models with compactified extra dimensions (Kaluza-Klein-type models), the main source of the photon time delay is the time variation of the electromagnetic coupling due to dimensional reduction, which induces an energy dependence of the speed of light. A similar relation between the fine-structure constant and the multidimensional gauge couplings also appears in models with large (noncompactified) extra dimensions. For photons of energies around $1 \mathrm{TeV}$ propagating at cosmological distances in an expanding universe, the time delay could range from a few seconds in the case of Kaluza-Klein models to a few days for models with large extra dimensions. As a consequence of the multidimensional effects, the intrinsic time profiles at the emitter rest frame differ from the detected time profiles. The formalism developed in the present paper allows the transformation of the predicted light curves of various energy ranges of the emitter into the frame of the observer for comparison with observations. Therefore, the study of the energy and redshift dependence of the time delay of photons emitted by astrophysical sources at cosmological distances could discriminate between the different multidimensional models and/or quantum gravity effects.
\end{abstract}

Subject headings: cosmology: theory — gamma rays: bursts — radiation mechanisms: general

\section{INTRODUCTION}

One of the most challenging issues of modern physics is the existence of extra dimensions, an idea proposed originally by Kaluza (1921) and developed by Klein (1926). Multidimensional geometries are the natural framework for the modern string/M theories (Witten 1996) or brane models (Horava \& Witten 1996). String models also provide a natural and selfconsistent explanation for the possible variation of the fundamental constants, as initially suggested by Dirac (1937, 1938 , 1979). Thus, the problem of the extra dimensions of the spacetime continuum is closely related to the problem of the variations of fundamental constants such as, e.g., the finestructure constant or the speed of light (for a recent review of experimental and theoretical studies and the present status of these fields, see Uzan 2003 and Magueijo 2003). Most theories with extra dimensions contain a built-in mechanism that allows variation of the fundamental constants. Within the multidimensional approach, the physical interactions are described by a theory formulated in $4+D$ dimensions and the conventional four-dimensional theory appears as a result of a process of dimensional reduction. Couplings in four dimensions are determined by a set of constants of the multidimensional theory and the size $A$ of the space of the extra dimensions. The multidimensional constants are assumed to be genuinely fundamental and consequently they do not vary with time. On the other hand, it is natural to assume that in an astrophysical or cosmological context $A$ varies with time, similar to the scale factor $a$ of our four-dimensional universe. However, this leads to the time variation in four dimensions of fundamental constants such as the fine-structure constant $\alpha$ or the gravitational coupling $G$. Moreover, since their time dependence is given by the same factor $A$, the time variations of $\alpha$ and $G$ could be correlated (Langacker et al. 2002).

The search for a unification of quantum mechanics and gravity is likely to require a drastic modification of the present-day deterministic representation of spacetime properties. There is at present no complete mathematical model for quantum gravity, and none of the many different models proposed so far can give a satisfactory description of the physics on characteristic scales near the Planck length $l_{\mathrm{P}}$. However, in several of the approaches that try to find a theory of quantum gravity the vacuum can acquire nontrivial optical properties because of gravitational recoil effects induced by the motion of the energetic particles. The recoil effects may induce a nontrivial refractive index, with photons at different energies traveling at different velocities (Ellis et al. 2000b). Photon polarization in a quantum spacetime may also induce birefringence (Gambini \& Pullin 1999), while stochastic effects in the vacuum could give rise to an energy-dependent diffusive spread in the velocities of different photons (Ellis et al. 2000c, 2000d).

Therefore, a large class of physical models incorporating quantum gravitation and/or multidimensional field theories predict that the propagation of a particle in a vacuum is modified by supplementary effects induced by modification of standard general relativity. In particular, the possible violation of the Lorentz invariance or the existence of extra dimensions can be investigated by studying the propagation of high-energy photons emitted by distant astrophysical sources (AmelinoCamelia et al. 1998).

The extragalactic gamma-ray sources of the highest energy in the known universe are the active galaxies called blazars: objects that emit jets of relativistic plasma aimed directly at us. Objects known as high-frequency BL Lac objects are expected to emit photons in the multi-TeV energy range. Only the nearest of these are expected to be observable in $\mathrm{TeV}$ energies, the others being hidden by intergalactic absorption (Stecker et al. 1996).

Extragalactic photons with the highest energy yet observed originated in a powerful flare coming from the giant elliptical active galaxy Mrk 501 in 1997 (Aharonian et al. 1997) and from Mrk 421 in 2001 (Krennrich et al. 2001). The high-energy 
flux of these emissions permitted detailed spectra to be extracted. Since as many as 25,000 photons were detected, the spectra were derived with a high statistical accuracy. The observation of the TeV photons from Mrk 501 and Mrk 421 allows us to impose some constraints on the quantum gravity scale and on the breaking of the Lorentz invariance (Biller et al. 1999; Stecker 2003). Quantum gravity phenomena are a result of quantum fluctuations on the Planck scale, $M_{\mathrm{P}}=$ $(\hbar c / G)^{1 / 2}$. In models involving large extra dimensions, such as, e.g., the brane-world models (Randall \& Sundrum 1999a, 1999 b), the energy scale at which gravity becomes strong can be much smaller than $M_{\mathrm{P}}$, with the quantum gravity scale $M_{\mathrm{QG}}$ approaching the $\mathrm{TeV}$ scale.

The data from the TeV gamma-ray flare associated with the active galaxy Mrk 421 have been used to place bounds on the possible energy dependence of the speed of light in the context of an effective quantum gravitational energy scale in Biller et al. (1999). The limits derived indicate that this energy scale is higher than $6 \times 10^{16} \mathrm{GeV}$ for the approach to quantum gravity in the context of D-brane string theory. By assuming a modified dispersion relation between the energy $E_{\gamma}$ and the momentum $p_{\gamma}$ of the photon of the form $E_{\gamma}^{2}=p_{\gamma}^{2}-p_{\gamma}^{3} / M_{\mathrm{QG}}$, and a similar relation for the electron, one can obtain the constraint $M_{\mathrm{QG}} \geq E_{\gamma}^{3} / 8 m_{e}^{2}$ for the quantum gravity scale (Stecker 2003). Since pair production occurs for energies of at least $20 \mathrm{TeV}$, it follows that $M_{\mathrm{QG}} \geq 0.3 M_{\mathrm{P}}$. The results also indicate an absence of evidence for violation of the Lorentz invariance, as proposed by some quantum gravity and multidimensional models.

Strong constraints on Lorentz-violating microscopic structures of spacetime such as, e.g., discreteness, noncommutativity, or extra dimensions, can be obtained from the observation of $100 \mathrm{MeV}$ synchrotron radiation from the Crab Nebula (Jacobson et al. 2003a). The Crab synchrotron emission has been observed to extend at least up to energies of $100 \mathrm{MeV}$, just before the Compton hump begins to contribute to the spectrum (Atoyan \& Aharonian 1996; Hillas et al. 1998). The magnetic field in the emission region has been estimated to be between 0.15 and $0.6 \mathrm{mG}$ (Hillas et al. 1998). The production of the observed radiation of $100 \mathrm{MeV}$ in this field requires a relativistic $\gamma$-factor of the order of $\gamma=3 \times 10^{9}$, corresponding to an electron energy of the order of $1500 \mathrm{TeV}$, with an electron velocity differing from $c$ by less then $10^{-19} c$. The observation of $100 \mathrm{MeV}$ synchrotron radiation from the Crab Nebula then gives the constraint $E_{\mathrm{QG}}>10^{26} \mathrm{GeV}$ (Jacobson et al. 2003b). Thus, this observation rules out this type of Lorentz violation, providing an important constraint on theories of quantum gravity, and imposes a stringent constraint on any modification of the dispersion relations of the electron that might be induced by quantum gravity.

The spectrum of the synchrotron radiation from the coupling of an electrically charged particle to an external magnetic field in the presence of quantum gravity effects of the general form $\left(E / M_{\mathrm{QG}}\right)^{\alpha}$ has been derived in Ellis et al. (2003b). The synchrotron constraint from the Crab Nebula practically excludes $\alpha \sim 1.74$ for $M_{\mathrm{QG}} \sim M_{\mathrm{P}} \sim 1.2 \times 10^{19} \mathrm{GeV}$. The model suggests a linear modification of the dispersion relation for the photon but not for the electron, and hence is compatible with known constraints from the Crab Nebula. New constraints on a possible Lorentz symmetry violation of the order of $E / M_{\mathrm{P}}$ for electrons and photons in the framework of effective field theory have been derived in Jacobson et al. (2003a) by using the absence of vacuum birefringence in the recently observed polarization of $\mathrm{MeV}$ emission from a gamma-ray burst (GRB) and the absence of vacuum Cerenkov radiation from the synchrotron electrons in the Crab Nebula. These constraints allow us to improve the previous bounds by 11 and 4 orders of magnitude, respectively.

The possibility of using of the high-energy radiation from gamma-ray pulsars to place limits on quantum gravity effects has been suggested by Kaaret (1999). The emission from the Crab pulsar at energies above $2 \mathrm{GeV}$ trails that at $70-100 \mathrm{MeV}$ by no more than $0.35 \mathrm{~ms}$ ( $95 \%$ confidence). This effect places a lower bound on the energy scale of quantum gravitational effects on the speed of light of $1.8 \times 10^{15} \mathrm{GeV}$. In the near future this bound might be improved by 2 orders of magnitude by observation of pulsations from the Crab at higher energies, of the order of 50-100 GeV.

The confirmation that at least some GRBs are indeed at cosmological distances raises the possibility that observations of these could provide interesting constraints on the fundamental laws of physics (for recent reviews on GRBs, see Zhang \& Meszaros 2004 and Cheng \& Lu 2001). The fine-scale time structure and hard spectra of GRB emissions are very sensitive to the possible dispersion of electromagnetic waves in vacuo, with velocity differences $\Delta u \sim E / E_{\mathrm{QG}}$, as suggested in some approaches to quantum gravity. GRB measurements might be sensitive to a dispersion scale $E_{\mathrm{QG}}$ comparable to the Planck energy scale $E_{\mathrm{P}} \sim 10^{19} \mathrm{GeV}$, sufficient to test some of these theories (Amelino-Camelia et al. 1998). Hence, the study of short-duration photon bursts propagating over cosmological distances is the most promising way to probe the quantum gravitational effects and/or the effects related to the existence of extra dimensions. The modification of the group velocity of the photons by the quantum effects would affect the simultaneity of the arrival times of photons with different energies. Thus, given a distant, transient source of photons one could measure the differences in the arrival times of sharp transitions in the signals in different energy bands. A key issue in such a probe is to distinguish the effects of the quantum gravity/ multidimensional medium from any intrinsic delay in the emission of particles of different energies by the source. The quantum gravity effects should increase with the redshift of the source, whereas source effects would be independent of the redshift in the absence of any cosmological evolution effects. Therefore, it is preferable to use transient sources with a known spread in redshift $z$. The best way to probe the time lags that might arise from quantum gravity effects is to use GRBs with known redshifts, which range up to $z \sim 5$.

Data on GRBs may be used to set limits on variations in the velocity of light. This has been illustrated by using BATSE and OSSE observations of the GRBs that have recently been identified optically and for which precise redshifts are available in Ellis et al. (2000a). A regression analysis can be performed to look for an energy-dependent effect that should correlate with redshift. The analysis of GRB data yields a limit $M_{\mathrm{QG}} \sim 10^{15} \mathrm{GeV}$ for the quantum gravity scale. The study of the times of flight of radiation from GRBs with known redshifts has been considerably improved by using a wavelet-shrinkage procedure for noise removal and a wavelet "zoom" technique to define with high accuracy the timings of sharp transitions in GRB light curves (Ellis et al. 2003a). This procedure optimizes the sensitivity of experimental probes of any energy dependence of the velocity of light. These wavelet techniques have been applied to $64 \mathrm{~ms}$ and time-tagged event data from BATSE, and also to OSSE data. A search for time lags between sharp transitions in GRB light curves in different energy bands yields the lower limit $M_{\mathrm{QG}} \geq 6.9 \times 10^{15} \mathrm{GeV}$ on the quantum 
gravity scale in any model with a linear dependence of the velocity of light, $c \sim E / M_{\mathrm{QG}}$.

High-energy GeV emissions from GRBs have already been detected (Sommer et al. 1994; Hurley 1994); there is also tentative evidence for $\mathrm{TeV}$ emissions (Atkins et al. 2000; Poirier et al. 2003). The production of $\mathrm{TeV}$ photons is also predicted by most of the GRB theories. The emission mechanisms for $\mathrm{TeV}$ photons include electron inverse Compton emission and synchrotron emission from the protons accelerated by GRB shocks. The shocks could be internal shocks, external forward shocks, or external reverse shocks of GRBs. Such very high energy photons at cosmological distances may largely be absorbed by interacting with the cosmic infrared background radiation (Madau \& Phinney 1996).

There are many astrophysical mechanisms that could produce a delay in the arrival time of high-energy photons or cosmic rays. The electron inverse scattering of the created electron-positron pairs of the cosmic microwave background photons will produce delayed $\mathrm{MeV}-\mathrm{GeV}$ emission. There are two likely mechanisms causing the time delay. One is the angular spreading effect of the secondary pairs, i.e., the scattered microwave photons deviate from the direction of the original $\mathrm{TeV}$ photons by an angle $\sim 1 / \gamma$, where $\gamma$ is the Lorentz factor of the electron-positron pairs (Cheng \& Cheng 1996; Dai \& Lu 2002; Wang et al. 2004). Another mechanism is related to the deflection of the direction of propagation of the pairs in the intergalactic magnetic field if this field is sufficiently strong (Plaga 1995). It is important to note that all these delay mechanisms predict that the low-energy photons come late. However, in the models with extra dimensions the high-energy photon comes later.

It is the purpose of the present paper to point out some other possible sources of time delay for high-energy photons from GRB emissions. Namely, we shall consider the effects of the existence of the extra dimensions on the propagation of highenergy photons in two distinct physical scenarios: KaluzaKlein theories with compactified extra dimensions and models with large extra dimensions. In Kaluza-Klein theories, the time variation of the scale factor of the space of extra dimensions leads to a time variation of both the gravitational constant $G$ and of the fine-structure constant $\alpha$. The variation of $\alpha$ is photon energy dependent, and via the time delay allows the estimation of the size of the (compactified) extra dimension. In the case of the large (noncompactified) extra dimensions, there is no multidimensional physical mechanism to induce a variation of $\alpha$. Recently, using high-resolution spectroscopy of QSO absorption spectra a time variation of $\alpha$ has been reported (Webb et al. 1999; Murphy et al. 2001, 2003). The detected rate of change of $\alpha$ is of the order $\Delta \alpha / \alpha \sim-10^{-5}$ at a redshift $z \sim 1.5$.

The present paper is organized as follows. In $\S 2$ we discuss the most important physical processes that could lead to the variation of the electromagnetic coupling in the different versions of the multidimensional models. The basic equations for the time delay of photons in Kaluza-Klein and RandallSundrum-type models are obtained in $\S 3$. In $\S 4$ we discuss and conclude our results.

\section{VARIATION OF THE ELECTROMAGNETIC COUPLING IN MODELS WITH EXTRA DIMENSIONS}

The starting point in the multidimensional approach of describing the fundamental interactions by Kaluza \& Klein is the consideration of the pure Einstein gravity in a multidimensional spacetime $M^{4} \times K^{D}$, described by the multidi- mensional metric tensor $\hat{g}_{M N}$. Here $M^{4}$ is the four-dimensional spacetime and $K^{D}$ is the compact manifold of extra dimensions. Generally, the reduced theory contains the Einstein gravity and the Yang-Mills fields, with the gauge group determined by the isometry group of the space of extra dimensions. The action of the multidimensional Kaluza-Klein theory is the action for the pure Einstein theory on $M^{4} \times K^{D}$, with the action given by (Overduin \& Wesson 1997)

$$
S=\frac{1}{16 \pi G_{(4+D)}} \int d^{4+D} \hat{x} \sqrt{-\hat{g}} R^{(4+D)},
$$

where $\hat{g}=\operatorname{det}\left(\hat{g}_{M N}\right), R^{(4+D)}$ is the scalar curvature in $M^{4} \times$ $K^{D}$, and $G_{(4+D)}$ is the multidimensional gravitational constant, which is assumed to be a true constant and does not depend on time. According to the procedure of dimensional reduction, the $(\mu, \nu=0,1,2,3)$ components of the metric tensor are identified as the four-dimensional metric tensor, while certain combinations of the rest of the components are identified as gauge-field multiplets $A_{\mu}$ and scalar fields $\phi_{m}, m=1,2, \ldots$. After the mode expansion of these fields, with the coefficients of the expansion (interpreted as four-dimensional fields) depending only on $x^{\mu}, \mu=0,1,2,3$, we obtain the fourdimensional action

$S_{0}=\int d^{4} x\left\{\frac{1}{16 \pi G(t)} R^{(4)}+\sum_{i} \frac{1}{4 g_{i}^{2}(t)} \operatorname{Tr}\left[F_{\mu \nu}^{(i)} F^{i(\mu \nu)}\right]\right\}$,

where $G(t) \equiv G_{4}(t)$ is the four-dimensional gravitational constant. In obtaining equation (2) we have considered only zero modes of the mode expansion. The parameters $g_{i}(t)$ are the gauge couplings, and the index $i$ labels the simple subgroups of the gauge group. The general reduction of the initial KaluzaKlein action $S$ also gives terms including nonzero modes of the gravitational, gauge, and scalar fields (Overduin \& Wesson 1997). The scalar fields give highly nonlinear interaction terms and are coupled nonminimally to the gravitational and gauge fields. In the following we neglect for simplicity the contribution of these scalar fields.

Identifying the gravitational and gauge couplings from the action $S_{0}$ for the zero modes, one obtains the following expressions for $G(t)$ and $g_{i}^{2}(t)$ in terms of $G_{(4+D)}$ and the radius $\Phi(t)$ of the space of the extra dimensions (Loren-Aguilar et al. 2003):

$$
G(t)=\frac{G_{(4+D)}}{V_{D}(t)}, \quad g_{i}^{2}(t)=\tilde{k}_{i} \frac{G_{(4+D)}}{\Phi^{2}(t) V_{D}(t)}
$$

where $V_{D}(t) \sim \Phi^{D}(t)$ is the volume of the space of the extra dimensions and $\tilde{k}_{i}$ are coefficients that depend on the isometry group of $K^{D}$. The fine-structure constant $\alpha(t)$ is given by linear combinations of $g_{i}^{2}(t)$, with the specific relation depending on the gauge group and the scheme of spontaneous symmetry breaking. Generally,

$$
\alpha(t)=k_{1} \frac{G_{(4+D)}}{\Phi^{2}(t) V_{D}(t)},
$$

where $k_{1}$ is a constant. Since $\dot{V}_{D}(t) / V_{D}(t)=d(\dot{\Phi} / \Phi)$, for the time variation of the fine structure constant we obtain

$$
\frac{\dot{\alpha}}{\alpha}=-(D+2) \frac{\dot{\Phi}}{\Phi} .
$$


The variation of the fine-structure constant in a more general model, in which a Yang-Mills-type field is also included in the $(4+D)$-dimensional spacetime, was also considered in Loren-Aguilar et al. (2003). The action is

$$
\begin{aligned}
S=\int d^{4+D} \hat{x} \sqrt{-\hat{g}}\left[\frac{1}{16 \pi G_{(4+D)}}\right. & R^{(4+D)} \\
& \left.+\frac{1}{4 g_{(4+D)}^{2}} \operatorname{Tr}\left(\hat{F}^{M N} \hat{F}_{M N}\right)\right],
\end{aligned}
$$

where $g_{(4+D)}^{2}$ is the multidimensional coupling, supposed to be constant in time. In this case, the dimensionally reduced theory includes the Einstein gravity and the four-dimensional gauge fields plus scalar fields with a quartic potential. The four-dimensional gravitational and fine-structure constants are given by

$$
G(t)=\frac{G_{(4+D)}}{V_{D}(t)}, \quad \alpha(t)=k_{2} \frac{G_{(4+D)}}{V_{D}(t)},
$$

where $k_{2}$ is a constant. The time variation of the fine-structure constant is

$$
\frac{\dot{\alpha}}{\alpha}=-D \frac{\dot{\Phi}}{\Phi} .
$$

We consider now models with large extra dimensions (brane-world models), as initially considered in Randall \& Sundrum (1999a). In the five-dimensional spacetime, the brane world is located at $Y\left(X^{I}\right)=0$, where $X^{I}, I=0,1,2,3,4$, are five-dimensional coordinates. The effective action in five dimensions is (Maeda \& Wands 2000; Chen et al. 2002)

$$
\begin{aligned}
S= & \int d^{5} X \sqrt{-g_{5}}\left(\frac{1}{2 k_{5}^{2}} R_{5}-\Lambda_{5}\right) \\
& +\int_{Y=0} d^{4} x \sqrt{-g}\left(\frac{1}{k_{5}^{2}} K^{ \pm}-\lambda+L^{\text {matter }}\right),
\end{aligned}
$$

where $k_{5}^{2}=8 \pi G_{5}$ is the five-dimensional gravitational coupling constant, $\Lambda_{5}$ is the cosmological constant in the bulk, $x^{\mu}, \mu=0,1,2,3$, are the induced four-dimensional braneworld coordinates, $R_{5}$ is the five-dimensional intrinsic curvature in the bulk, and $K^{ \pm}$is the extrinsic curvature on either side of the brane.

Assuming a metric of the form $d s^{2}=\left(n_{I} n_{J}+g_{I J}\right) d x^{I} d x^{J}$, with $n_{I} d x^{I}=d \chi$ the unit normal to the $\chi=$ constant hypersurfaces and $g_{I J}$ the induced metric on $\chi=$ constant hypersurfaces, the effective four-dimensional gravitational equations on the brane take the form (Shiromizu et al. 2000; Sasaki et al. 2000; Chen et al. 2001a, 2001b)

$$
G_{\mu \nu}=-\Lambda g_{\mu \nu}+k_{4}^{2} T_{\mu \nu}+k_{5}^{4} S_{\mu \nu}-E_{\mu \nu}
$$

where

$$
S_{\mu \nu}=\frac{1}{12} T T_{\mu \nu}-\frac{1}{4} T_{\mu} T_{\nu \alpha}+\frac{1}{24} g_{\mu \nu}\left(3 T^{\alpha \beta} T_{\alpha \beta}-T^{2}\right),
$$

$\Lambda=k_{5}^{2}\left(\Lambda_{5}+k_{5}^{2} \lambda^{2} / 6\right) / 2$, and $k_{4}^{2}=k_{5}^{4} \lambda / 6$. Here $E_{I J}=C_{I A J B} n^{A} n^{B}$, where $C_{I A J B}$ is the five-dimensional Weyl tensor in the bulk, $\lambda$ is the vacuum energy on the brane, $T_{\mu \nu}$ is the matter energy- momentum tensor on the brane, and $T=T^{\mu}{ }_{\mu}$ is the trace of the energy-momentum tensor.

The reduction formula expressing the four-dimensional Planck mass $M_{\mathrm{P}}$ in terms of the fundamental (five-dimensional) mass scale $M=\left(16 \pi \hat{G}_{(5)}\right)^{-1 / 3} \sim k$ has been derived in Boos et al. (2002). The result is

$$
M_{\mathrm{P}}^{2}=\frac{M^{3}}{k}\left(e^{2 \pi k \Phi}-1\right) .
$$

Since in the models with large extra dimensions all matter fields are localized on the brane and do not depend on the radius of the extra dimension, there is no simple mechanism for the variation of the fine-structure constant. A possible form of the variation of $\alpha$ has been obtained in Loren-Aguilar et al. (2003), giving

$$
\alpha(t)=k_{3} \frac{g_{(5)}^{2}}{\Phi(t)}, \quad \frac{\dot{\alpha}}{\alpha}=-\frac{\dot{\Phi}}{\Phi},
$$

where $k_{3}$ is a constant.

Finally, we briefly consider the problem of the quantum corrections to the expressions for the fine-structure constant presented above. This question has been discussed in Langacker et al. (2002) and Loren-Aguilar et al. (2003). To relate the value of $\alpha$ obtained at the scale $M_{\Phi}=\Phi^{-1}(t)$ to its value at some low-energy scale $\mu$ by taking into account quantum corrections, one must use the renormalization group formulae for running couplings, which gives for the time variation of $\alpha$

$$
\frac{\dot{\alpha}(\mu, t)}{\alpha(\mu, t)}=\frac{\dot{\alpha}\left(M_{\Phi}, t\right)}{\alpha\left(M_{\Phi}, t\right)}-\alpha(\mu, t) A \frac{\dot{\Phi}}{\Phi}(1+\ln \mu \Phi),
$$

where $A$ is a constant of the order of 1 .

The second term in equation (14) is of the order of $O(\alpha)$. Hence, it is a subdominant term, thus showing that quantum effects do not significantly affect the time variation of the fine-structure constant.

\section{TIME DELAY OF PHOTONS IN MULTIDIMENSIONAL EXPANDING UNIVERSES}

We consider first the propagation of gamma-rays from GRBs in the Kaluza-Klein-type models. For simplicity we restrict our discussion to the five-dimensional case. Hence, we assume a flat Friedmann-Robertson-Walker-type background metric of the form

$$
\begin{aligned}
d s^{2}= & c^{2} d t^{2}-a^{2}(t)\left[d r^{2}+r^{2}\left(d \theta^{2}+\sin ^{2} \theta d \varphi^{2}\right)\right] \\
& +\varepsilon \Phi^{2}(t) d v^{2}
\end{aligned}
$$

where $a$ is the scale factor of the universe, $\varepsilon= \pm 1$, and $\Phi$ is the scale factor of the fifth dimension, denoted by $v$. We also assume that the time variation of the fine-structure constant is entirely due to the change in the speed of light $c$. Therefore, we neglect any possible time variation of the electric charge or Planck's constant. Then the time variation of the speed of light can be related to the size of the fifth dimension by means of the general equation

$$
\frac{\Delta \dot{c}}{\Delta c}=\beta \varepsilon \frac{\dot{\Phi}}{\Phi}
$$


where $\beta=1$ in the case of the Einstein-Yang-Mills model and $\beta=3$ for the case of the pure Einstein gravity in five dimensions. Equation (16) can be integrated to give

$$
c=c_{0}\left(1+\varepsilon \Phi^{\beta}\right)
$$

where $c_{0}$ is an arbitrary integration constant. In order to find a simple and directly testable relation between the radius of the extra dimension and the energy of the photon we shall assume, following the initial proposal in Ma (1990a, 1990b), that the mass of a body (and the associated energy) corresponds to the length of a "line segment" of the fifth subspace. Such a relation embodies the spirit of Mach's principle in the sense that the inertial mass depends on the distribution of matter in the universe. In a more general formulation, we assume that the variables parameters $c, G$, and the photon energy $E=h \nu$ are related to the metric tensor component of the fifth dimension by means of the equation (Mak \& Harko 1999)

$$
\frac{G(t) E}{c^{4}}=\frac{1}{\gamma} \int_{v^{0}}^{v} \sqrt{\left|g_{44}\right|} d v=\frac{1}{\gamma} \int_{v^{0}}^{v} \Phi d v
$$

where $\gamma$ is an arbitrary constant.

If $\Phi$ is independent of $v$, as is the case in models with compactified extra dimensions, equation (18) gives

$$
\Phi=\frac{\gamma G E}{c^{4}\left(v-v^{0}\right)}=\frac{E}{E_{\mathrm{K}}},
$$

where we denoted $E_{\mathrm{K}}=c^{4} \Delta v / \gamma G$, with $\Delta v=v-v^{0}$ describing the variation of the size of the fifth dimension between the moments of the emission and detection of a photon. Therefore, the energy dependence of the speed of light of the photon is given by

$$
c=c_{0}\left[1+\varepsilon\left(\frac{E}{E_{\mathrm{K}}}\right)^{\beta}\right] .
$$

We consider two photons emitted during a GRB with presentday energies $E_{1}$ and $E_{2}$. At earlier epochs, their energies would have been blueshifted by a factor of $1+z$. It thus follows that the difference in the velocities of the two photons is given by

$$
\Delta c=c_{0} \frac{\Delta E(1+z)}{E_{\mathrm{K}}}, \quad \beta=1,
$$

and

$$
\Delta c=c_{0} \frac{f\left(E_{1}, E_{2}\right)(1+z)^{3}}{E_{\mathrm{K}}^{3}}, \quad \beta=3,
$$

respectively, where we denoted $\Delta E=E_{1}-E_{2}$ and $f\left(E_{1}, E_{2}\right)=$ $E_{1}^{3}-E_{2}^{3}$. A linear energy dependence of the difference of the photon velocities has also been considered in Ellis et al. (2003a) as a result of the dispersion-relation analysis of the Maxwell equations in the nontrivial background metric perturbed by the recoil of a massive spacetime defect during the scattering of a low-energy photon or neutrino.
For light propagating from cosmological distances, the differential relation between time and redshift is (Ellis et al. 2003a)

$$
d t=-H_{0}^{-1} \frac{d z}{(1+z) h(z)}
$$

where

$$
g(z)=\sqrt{\Omega_{\Lambda}+\Omega_{M}(1+z)^{3}},
$$

$H_{0}=72 \mathrm{~km} \mathrm{~s}^{-1} \mathrm{Mpc}^{-1}$ (Freedman et al. 2001), and $\Omega_{M} \approx 0.3$ and $\Omega_{\Lambda} \approx 0.7$ are the mass density parameter and the dark energy parameter, respectively (Peebles \& Ratra 2003). A particle with a velocity $c$ travels an elementary distance $c d t=-H_{0}^{-1} c d z /(1+z) g(z)$, with the difference $\Delta L$ in the distances covered by the two particles given by $\Delta L=$ $H_{0}^{-1} \int_{0}^{z} \Delta c d z /(1+z) g(z)$ (Ellis et al. 2003a). By taking into account the expression for $\Delta c$, we obtain the following equations describing the time delay of two photons:

$$
\begin{gathered}
\Delta t=H_{0}^{-1} \frac{\Delta E}{E_{\mathrm{K}}} \int_{0}^{z} \frac{d z}{g(z)}, \quad \beta=1, \\
\Delta t=H_{0}^{-1}\left[\frac{f\left(E_{1}, E_{2}\right)}{E_{\mathrm{K}}}\right]^{3} \int_{0}^{z} \frac{(1+z)^{2} d z}{g(z)}, \quad \beta=3 .
\end{gathered}
$$

In the case of isotropic homogeneous cosmological models with large extra dimensions, there is a nonzero contribution from the five-dimensional Weyl tensor from the bulk, expressed by a scalar term $U$, called dark radiation (Chen et al. 2002; Harko \& Mak 2003; Chen et al. 2003). The dark radiation term is a pure bulk (five-dimensional) effect, and therefore we cannot determine its expression without solving the complete system of field equations in five dimensions. In the case of a Friedmann-Roberston-Walker-type cosmological model, the expression of the dark radiation is (Chen et al. 2001a; Dabrowski et al. 2002)

$$
U=\frac{U_{0}}{a^{4}}
$$

with $U_{0}$ an arbitrary constant of integration. Since the fifth dimension is large, the scale factor $\Phi$ can also be a function of $v$. Hence, an explicit knowledge of the $v$-dependence of $\Phi$ is needed in order to derive the speed of light-photon energy dependence. However, taking into account equation (13), which shows a linear dependence of $\alpha$ on the scale of the fifth dimension, we can assume that the time delay between two different energy photons emitted during a GRB is given by

$$
\Delta t=H_{0}^{-1} \frac{\Delta E}{E_{F}} \int_{0}^{z} \frac{d z}{h(z)},
$$

where $E_{F}$ is the energy scale associated with the large extra dimensions and

$$
h(z)=\sqrt{\Omega_{\Lambda}+\Omega_{M}(1+z)^{3}+\Omega_{U}(1+z)^{4}},
$$

with $\Omega_{U}$ the dark radiation parameter. 


\section{DISCUSSIONS AND FINAL REMARKS}

In order to calculate the delay in the gamma-ray photons' arrival time in the Kaluza-Klein-type models, we need to estimate first the Kaluza-Klein energy scale $E_{\mathrm{K}}=c^{4} \Delta v / \gamma G$ for which the effects from the extra dimensions become important. Assuming that the size and the variation of the extra dimension $\Delta v$ is of the same order as the Planck length, $\Delta v=l_{\mathrm{P}}=$ $1.6 \times 10^{-33} \mathrm{~cm}$, the Kaluza-Klein energy scale is equal, for $\gamma=1$, to $E_{\mathrm{K}}=1.2 \times 10^{19} \mathrm{GeV}$. Of course, a large value of $\gamma$ can decrease the Kaluza-Klein energy scale. The variation of the difference in the photon arrival time for different values of the photon energy is presented in Figure 1 for the EinsteinYang-Mills model corresponding to $\beta=1$ and with $\gamma=1$.

Because of the power of 3 energy dependence of the time delay in the pure Einstein gravity model $(\beta=3)$, the value of $\Delta t$ is extremely small for $\gamma=1$, corresponding to the $E_{\mathrm{K}}=1.2 \times 10^{19} \mathrm{GeV}$ energy scale. In this case $\Delta t \approx 10^{-30} \mathrm{~s}$ even for photon energies of the order of $1 \mathrm{TeV}$. In order to obtain some observable effects a very large value of $\gamma$, of the order of $\gamma=10^{10}$, is required. The variation of the photon time delay in the case of the Kaluza-Klein compactified model with pure Einstein gravity is represented in Figure 2.

The analysis of the BATSE and OSSE data has imposed a lower limit $E_{\mathrm{QG}} \geq 6.9 \times 10^{15} \mathrm{GeV}$ on the quantum gravity scale in the linear model (Ellis et al. 2003a), which is much smaller than the Kaluza-Klein energy scale we have considered. If the fundamental energy scale is of the order of $E_{\mathrm{OG}}$, then the time delay between $\mathrm{TeV}$ and $\mathrm{keV} / \mathrm{MeV}$ photons could have larger values than those considered in the present approach.

To generate the correct hierarchy between the Planck scale and the $\mathrm{TeV}$ scale in models with large extra dimensions, the product $k \Phi$ must be of the order of $k \Phi \approx 11-12$ (Boos et al. 2002). There are no simple mechanisms to describe the temporal evolution of the fine-structure constant in this type of model, a variation of $\alpha$ requiring the consideration of bulk gauge and, perhaps, fermionic fields. However, based on the analogy with the Kaluza-Klein case one can assume a linear dependence of the time delay on $\Delta E$, with the characteristic energy scale $E_{F}$ a parameter to be determined from observations. The variation of the time delay for two photons of

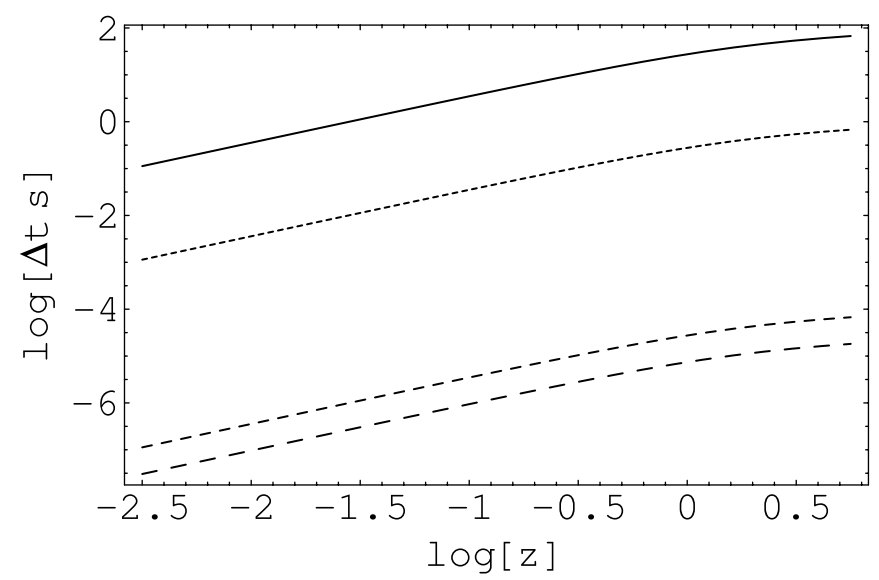

FIG. 1.-Variation of the photon time delay as a function of the redshift $z$ (in a logarithmic scale) in the compact Kaluza-Klein model, with a EinsteinYang-Mills-type action $(\beta=1)$ for $\gamma=1$ (corresponding to a fundamental energy scale $E_{\mathrm{K}}=1.2 \times 10^{19} \mathrm{GeV}$ ) and for different photon energy values: solid line, $E_{1}=1 \mathrm{TeV}, E_{2}=1 \mathrm{eV}$; dotted line, $E_{1}=10 \mathrm{GeV}, E_{2}=1 \mathrm{MeV}$; short-dashed line, $E_{1}=1 \mathrm{MeV}, E_{2}=1 \mathrm{eV}$; long-dashed line, $E_{1}=300 \mathrm{keV}$, $E_{2}=30 \mathrm{keV}$. For the mass and dark energy parameters, we have used the values $\Omega_{M}=0.3$ and $\Omega_{\Lambda}=0.7$, respectively.

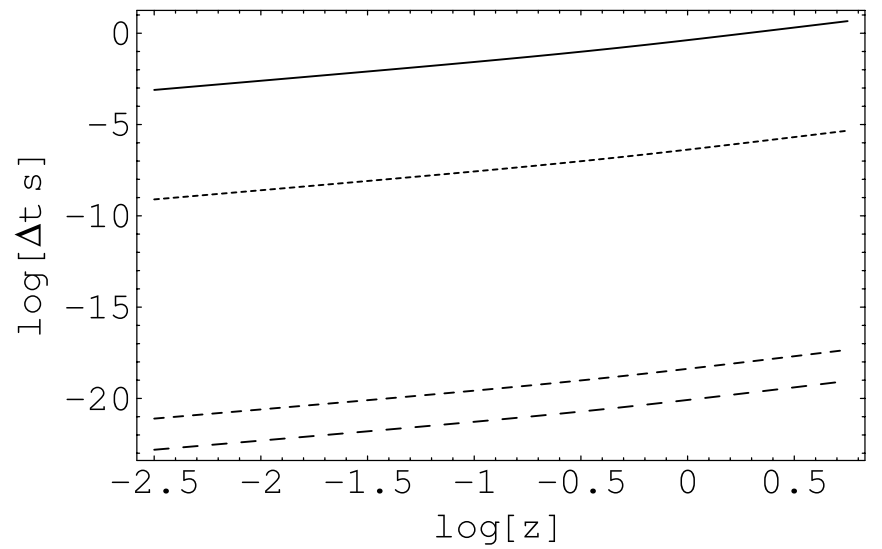

Fig. 2.-Variation of the photon time delay as a function of the redshift $z$ (in a logarithmic scale) in the compact Kaluza-Klein model with a pure Einstein-type action $(\beta=3)$ for $\gamma=10^{10}$ (corresponding to a fundamental energy scale $E_{\mathrm{K}}=1.2 \times 10^{9} \mathrm{GeV}$ ) and for different photon energy values: solid line, $E_{1}=1 \mathrm{TeV}, E_{2}=1 \mathrm{eV}$; dotted line, $E_{1}=10 \mathrm{GeV}, E_{2}=1 \mathrm{MeV}$; short-dashed line, $E_{1}=1 \mathrm{MeV}, E_{2}=1 \mathrm{eV}$; long-dashed line, $E_{1}=300 \mathrm{keV}$, $E_{2}=30 \mathrm{keV}$. For the mass and dark energy parameters, we have used the values $\Omega_{M}=0.3$ and $\Omega_{\Lambda}=0.7$, respectively.

different energy is represented for $E_{F}=7 \times 10^{15} \mathrm{GeV}$ in Figure 3.

In deriving the equations for the time delay due to the presence of the effects of the extra dimensions we have used a crucial assumption, namely that there is an intrinsic synchronization of pulsing emission of photons at different energy ranges. This assumption allows the consistent determination, by comparison with observations, of the parameters determining the effects of the extra dimensions on the photon propagation. However, if the emission of the photons of different energies is not synchronized, the simple processing of data for the delay of the photons is not enough to constrain the multidimensional effects. There are many proposed explanations that could produce a delayed timescale at the emitter between, e.g., $\mathrm{GeV}$ and $\mathrm{keV} / \mathrm{MeV}$ photons, as observed in the case of GRB 940217 (Hurley 1994). These explanations include interaction of $\mathrm{TeV}$ photons with cosmic infrared

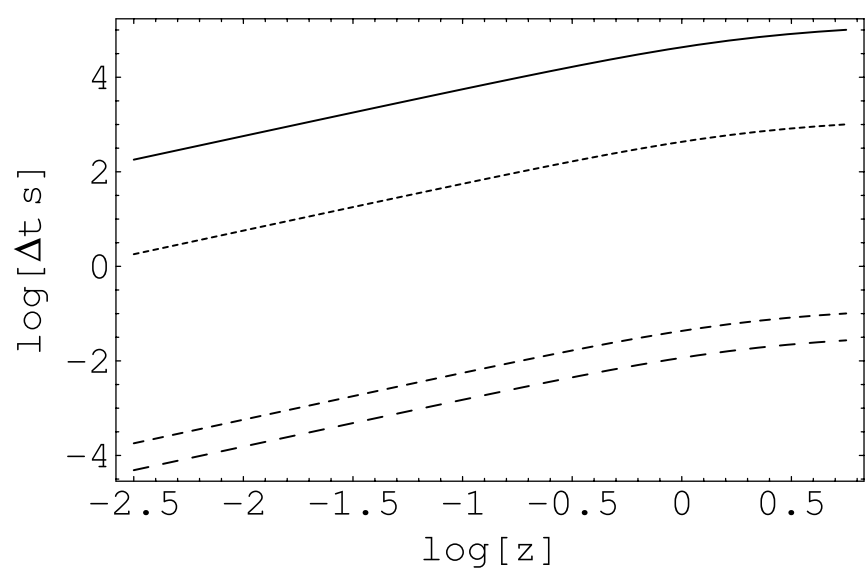

FIG. 3.-Variation of the photon time delay as a function of the redshift $z$ (in a logarithmic scale) in cosmological models with large extra dimensions for a fundamental energy scale $E_{F}=7 \times 10^{15} \mathrm{GeV}$ and for different photon energy values: solid line, $E_{1}=1 \mathrm{TeV}, E_{2}=1 \mathrm{eV}$; dotted line, $E_{1}=10 \mathrm{GeV}$, $E_{2}=1 \mathrm{MeV}$; short-dashed line, $E_{1}=1 \mathrm{MeV}, E_{2}=1 \mathrm{eV}$; long-dashed line, $E_{1}=300 \mathrm{keV}, E_{2}=30 \mathrm{keV}$. For the mass, dark energy, and dark radiation parameters we have used the values $\Omega_{M}=0.3, \Omega_{\Lambda}=0.68$, and $\Omega_{U}=0.02$, respectively. 

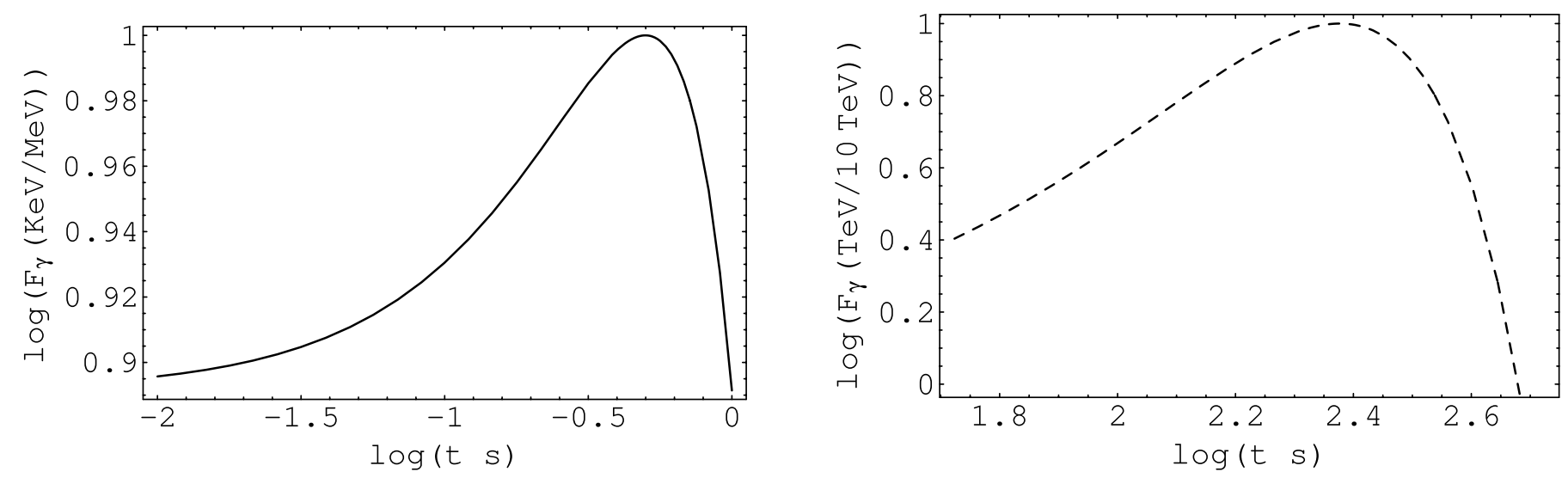

FIG. 4.-Comparison, in arbitrary units, of the initial keV/MeV time profile of the GRB emission occurring at a redshift $z=3$ (assumed to have a Gaussian

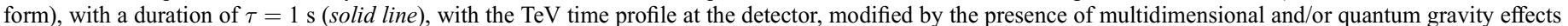

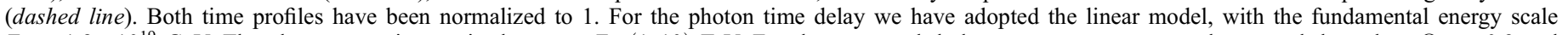

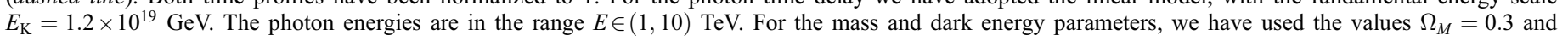
$\Omega_{\Lambda}=0.7$, respectively.

background photons (Plaga 1995), interactions of ultrarelativistic protons with a dense cloud (Katz 1994), or inverse Compton scattering in early forward and reverse shocks (Meszaros \& Rees 1994). In these cases, in order to consistently determine the time delay between photons at different energies due to multidimensional propagation effects the knowledge of the initial time profiles of $\mathrm{MeV}, \mathrm{GeV}$, and $\mathrm{TeV}$ photons is also required. The description of the nonsynchronized-emission time profiles is also model dependent, and thus in this case it is difficult to clearly distinguish between emission and propagation effects. However, despite all these possible methods of producing a time delay of different energy photons at the emitter, in our model we make two very clear predictions, namely (1) that the high-energy (TeV) photons have the higher delay and (2) that the delay timescales are correlated with the redshift $z$ of the emitting source, because the delay results from propagation effects. In the future, if enough observational data become available, the study of the energy and redshift dependence of the delay $\Delta t=\Delta t\left(E_{\gamma}, z\right)$ could lead to the possibility of discriminating between emission and propagation effects.

We also suggest that if there is an intrinsic synchronization of the photon emission at different energies, then because of the effects of the extra dimensions the detected time profiles between the $\mathrm{keV} / \mathrm{MeV} / \mathrm{GeV}$ and the $\mathrm{TeV}$ bursts should be very different. We want to emphasize that it is better to measure the time profile difference between $\mathrm{keV} / \mathrm{MeV}$ and $\mathrm{TeV}$ photons instead of the difference between $\mathrm{keV} / \mathrm{MeV}$ and $\mathrm{GeV}$ photons, in order to avoid contamination or other effects. For an emitter with an initial Gaussian time profile $e^{-(t / \tau)^{2}}$, where $\tau$ is the duration of the burst, the pulse shape of the $1-10 \mathrm{TeV}$ photons is shown in Figure 4. We have considered that the GRB occurred at a redshift of $z=3$, and considered the initial duration of the burst $\tau=1 \mathrm{~s}$. For the time delay of the photons we have adopted the linear model, with the fundamental energy scale of the same order of magnitude as the Planck scale, $E_{\mathrm{K}}=$ $1.2 \times 10^{19} \mathrm{GeV}$. In this case the time delays of the TeV photons with respect to the $\mathrm{keV} / \mathrm{MeV} / \mathrm{GeV}$ photons are $\Delta t_{1}=52.9 \mathrm{~s}$ for the $1 \mathrm{TeV}$ photon and $\Delta t_{2}=529.3 \mathrm{~s}$ for the $10 \mathrm{TeV}$ photon, respectively.

Realistically, in order to determine the exact amount of delay time we have to measure the time profiles at the detector of both the $\mathrm{keV} / \mathrm{MeV}$ and of the $\mathrm{TeV}$ bursts, respectively. If the time delay between the $\mathrm{keV} / \mathrm{MeV}$ bursts and $\mathrm{TeV}$ bursts is longer than several seconds, the ground-based TeV telescopes are capable of catching the burst. A good coordinate effort between the Swift satellite and the ground-based TeV telescopes can easily make this measurement possible (Weekes 2003).

Observations of the time delay in GRBs have been proposed up to now mainly as tests of quantum gravity effects. In a pioneering work, Amelino-Camelia et al. (1998) suggested that quantum gravitational effects could induce a deformed dispersion relation for photons of the form $c^{2} \boldsymbol{p}^{2}=E^{2}[1+$ $\left.f\left(E / E_{\mathrm{QG}}\right)\right]$. By representing $f$ in the form of a power series, the energy-dependent velocity of the speed of light can be represented as $v \approx c\left(1 \pm E / E_{\mathrm{QG}}\right)$. This type of velocity dispersion results from a picture of the vacuum as a quantum gravitational "medium" that responds differently to the propagation of particles of different energies. This is analogous to propagation through a conventional medium, such as an electromagnetic plasma. The gravitational medium is generally believed to contain microscopic quantum fluctuations, which may occur on scale sizes of the order of the Planck length. In this approach, the vacuum is viewed as a nontrivial medium containing "foamy" quantum gravity fluctuations. The nature of this foamy vacuum may be visualized, for example, by imagining processes that include the pair creation of virtual black holes. The light propagation in the semiclassical spacetime that emerges in canonical quantum gravity in the loop representation was considered by Gambini \& Pullin (1999). In such a picture, spacetime exhibits a polymer-like structure at microscopic scales and departures from the perfect nondispersiveness of an ordinary vacuum naturally occur. Maxwell equations are modified by the quantum gravity, and nonvanishing corrections to the electromagnetic field equations appear that depend on the helicity of the propagating waves. These effects could lead to constraints on the discrete nature of the quantum spacetime from the study of GRBs at different energies.

In the present paper we have considered a different class of effects that could generate a time delay $\Delta t$ of the high-energy photons emitted during the GRBs, namely the possibility that the extra dimensions of the spacetime may modify the speed of light in vacuum. This is mainly due to the dependence of the fine-structure constant $\alpha$ on the extra dimensions. The effects related to extra dimensions in the variation of $\alpha$ are much 
stronger than the quantum gravity effects. In models with extra dimensions, the speed of light-photon energy dependence can be obtained exactly by considering the background gravitational field and the scalar and Yang-Mills-type gauge fields in extra dimensions. In quantum gravity models, this dependence is modeled more or less phenomenologically. On the other hand, the observation of the time delay of the photons in GRBs could provide some astrophysical tests to confirm the existence of extra dimensions of spacetime.

We have analyzed in detail the time delay for models with both compact and noncompact extra dimensions, deriving some explicit expressions for $\Delta t$. In the case of Kaluza-Kleintype theories, the compactification of extra dimensions provides a natural framework for the variation of the fine-structure constant and for the speed of light. The basic energy scale for this model is of the order of $10^{19} \mathrm{GeV}$, which for EinsteinYang-Mills-type models gives a delay that can be as high as $\Delta t=10^{2} \mathrm{~s}$ for $1 \mathrm{TeV}$ photons emitted at a redshift of $z=5$. In these models, the variation in the fine-structure constant is dominated by the effects of the extra dimensions and the quantum effects can be neglected.

Since in models with large extra dimensions the energy scale can be reduced significantly as a function of the size of $v$, much higher time delays are expected, which could be of the order of $10^{5} \mathrm{~s}$ for $1 \mathrm{TeV}$ photons. There is a strong model dependence of the Kaluza-Klein-type time delay expressions, the results depending on the initial (multidimensional) field structure. For a pure Einstein gravity in higher dimensions the photon time delay has extremely small values, which make it extremely difficult to detect.

For the detection of quantum gravity or extra dimensional effects, GRBs offer the most reliable high-energy photon sources located at cosmological distances. The BATSE data have already been used to extract valuable information on the quantum gravity energy scale (Ellis et al. 2003a). However, these data are restricted to a low energy range, of the order of $30-300 \mathrm{keV}$. In this range other concurrent physical processes, such as Compton scattering, could reduce the effect. In order to obtain more reliable data, a significant increase in the detected photon energy is necessary. The detection of quantum gravitational and extra dimensional features would also require the correlation of GRB redshifts with the temporal and energetic signatures.

The discovery of the linear polarization of the gamma-rays from the GRBs, with the estimated degree of polarization of $80 \% \pm 20 \%$, very close to the absolute maximum of $100 \%$, provides another test of quantum gravity effects (Mitrofanov 2003). If the effects of quantum gravity are linearly proportional to the ratio $E / E_{\mathrm{QG}}$, then the polarization of photons with energies of $\sim 0.1 \mathrm{MeV}$ should be completely random, contrary to the observations. Consequently, quantum gravity effects act with a power greater than 1 . The linear polarization of gamma rays also allows us to test the birefringence property of the quantum vacuum, as suggested by the quantum-gravity-inloop representation (Gambini \& Pullin 1999). Because of this property, two photons with opposite states of helicity have different group velocities (Gleiser \& Kozameh 2001;
Mitrofanov 2003). A significant rotation of the plane of polarization of a linearly polarized photon must occur long before any difference in time of arrival is even measurable. If a rotation, if present, is below a certain bound, one can obtain a general bound on the model parameters characterizing the effect (Gleiser \& Kozameh 2001). By analyzing the presence of linear polarization in the optical and ultraviolet spectrum of some distant sources, the limit $\chi<5 \times 10^{-5}$ has been found for the dimensionless parameter $\chi$ that characterizes both parity nonconservation and violation of Lorentz invariance (Gleiser $\&$ Kozameh 2001). This upper bound on $\chi$ induces a time delay of the order of $10^{-9} \mathrm{~s}$, which is beyond the possibility of observation. However, for cosmological GRBs, located at a distance of around $10^{10} \mathrm{lt}-\mathrm{yr}$, the quadratic birefringence of quantum spacetime could be tested by polarization measurements of photons with energies greater than $100 \mathrm{MeV}$ (Mitrofanov 2003). On the other hand, in models with extra dimensions there is a third-order dependence on energy of the speed of light, which could also be tested by using polarimetry of gamma-rays from cosmological sources. The combinations of both approaches based on time-delay measurements and polarimetry could provide significant constraints on quantum gravity and multidimensional models.

Ground-based atmospheric Cerenkov telescopes offer a unique opportunity for the observation of the delayed $\mathrm{TeV}$ components of GRBs. In the past few years, such telescopes using the Cerenkov imaging technique have proved to be remarkably sensitive for the detection of sources with hard gamma-ray spectra (Weekes et al. 2002; Weekes 2003). Because of the very large collection areas associated with these telescopes $\left(>50,000 \mathrm{~m}^{2}\right)$, they are particularly sensitive to the detection of transients, e.g., from active galactic nuclei such as Mrk 421 (Gaidos et al. 1996). These telescopes are more sensitive than the all-sky-viewing ground-level particle detectors such as Milagro, the Tibet array, or the Astrophysical Radiation and Gamma Observatory (ARGO), but have limited fields of view. However, they have been used in attempts to detect $\mathrm{TeV}$ emission from classical GRBs (Connaughton et al. 1997) and from primordial black holes (Connaughton et al. 1998). Although the detectable fluence can be as little as $10^{-8} \mathrm{ergs} \mathrm{s}^{-1}$, no detections have been reported yet. However, in no instance has a GRB been reported within the field of view of an operating imaging atmospheric Cerenkov telescope. Reported observations have been limited by the slew time of the telescope, the uncertainty in the initial source position, and the limited time of operation.

Therefore, the detection of the time delay between Tev and $\mathrm{GeV} / \mathrm{MeV}$ photons from GRBs could represent a new possibility for the study and understanding of some fundamental aspects of the physical laws governing our universe.

This work is supported by an RGC grant of the Hong Kong Government (project HKU 7014/04P). The authors would like to thank Professor T. C. Weekes, Professor Z. G. Dai, Y. F. Huang, X. Y. Wang, and the anonymous referee for suggestions that significantly improved the manuscript.

\section{REFERENCES}

Aharonian, F. A., et al. 1997, A\&A, 327, L5

Amelino-Camelia, G., Ellis, J., Mavromatos, N. E., Nanopoulos, D. V., \& Sarkar, S. 1998, Nature, 393, 763

Atkins, R., et al. 2000, ApJ, 533, L119

Atoyan, A. M., \& Aharonian, F. A. 1996, MNRAS, 278, 525
Biller, S. D., et al. 1999, Phys. Rev. Lett., 83, 2108

Boos, E. E., Kubyshin, Y. A., Smolyakov, M. N., \& Volobuev, I. P. 2002, Classical Quantum Gravity, 19, 4591

Chen, C. M., Harko, T., Kao, W. F., \& Mak, M. K. 2002, Nucl. Phys. B, 636, 159 
Chen, C. M., Harko, T., Kao, W. F., \& Mak, M. K. 2003, J. Cosmology Astropart. Phys., 11, 5

Chen, C. M., Harko, T., \& Mak, M. K. 2001a, Phys. Rev. D, 64, 044013 2001b, Phys. Rev. D, 64, 124017

Cheng, K. S., \& Lu, T. 2001, Chinese J. Astron. Astrophys., 1, 1

Cheng, L. X., \& Cheng, K. S. 1996, ApJ, 459, L79

Connaughton, V., et al. 1997, ApJ, 479, 859 . 1998, Astropart. Phys., 8, 179

Dabrowski, M. P., Godlowski, W., \& Szydlowski, M. 2002, preprint (astro-ph/ 0212100)

Dai, Z. G., \& Lu, T. 2002, ApJ, 580, 1013

Dirac, P. A. M. 1937, Nature, 139, 323

1938, Proc. R. Soc. London A, 165, 198

1979, Proc. R. Soc. London A, 365, 19

Ellis, J., Farakos, K., Mavromatos, N. E., Mitsou, V., \& Nanopoulos, D. V. 2000a, ApJ, 535, 139

Ellis, J., Mavromatos, N. E., \& Nanopoulos, D. V. 2000b, Phys. Rev. D, 61,027503

2000c, Gen. Relativ. Gravitation, 32, 127

2000d, Phys. Rev. D, 62, 084019

Ellis, J., Mavromatos, N. E., Nanopoulos, D. V., \& Sakharov, A. S. 2003a, A\&A, 402, 409

Ellis, J., Mavromatos, N. E., \& Sakharov, A. S. 2003b, Astropart. Phys., 20, 669

Freedman, W. L., et al. 2001, ApJ, 553, 47

Gaidos, J. A., et al. 1996, Nature, 383, 319

Gambini R., \& Pullin, J. 1999, Phys. Rev. D, 59, 124021

Gleiser R. J., \& Kozameh, C. N. 2001, Phys. Rev. D, 64, 083007

Harko, T., \& Mak, M. K. 2003, Classical Quantum Gravity, 20, 407

Hillas A. H., et al. 1998, ApJ, 503, 744

Horava, P., \& Witten, E. 1996, Nucl. Phys. B, 475, 94

Hurley, K. 1994, Nature, 372, 652

Jacobson, T., Liberati, S., \& Mattingly, D. 2003a, Nature, 424, 1019

Jacobson, T., Liberati, S., Mattingly, D., \& Stecker, F. W. 2003b, preprint (astro-ph/0309681)

Kaaret, P. 1999, A\&A, 345, L32

Kaluza, T. 1921, Sitzungsberichte Preussische Akademie der Wissenschaften, 96,69
Katz, J. I. 1994, ApJ, 432, L27

Klein, O. 1926, Zeitschrift fur Physik, 37, 895

Krennrich, F., et al. 2001, ApJ, 560, L45

Langacker, P., Segre, G., \& Strassler, M. J. 2002, Phys. Lett. B, 528, 121

Loren-Aguilar, P., Garcia-Berro, E., Isern, J., \& Kubyshin, Y. A. 2003, Classical Quantum Gravity, 20, 3885

Ma, G. W. 1990a, Phys. Lett. A, 146, 375 1990b, Phys. Lett. A, 143, 183

Madau, P., \& Phinney, E. S. 1996, ApJ, 456, 124

Maeda, K., \& Wands, D. 2000, Phys. Rev. D, 62, 124009

Magueijo, J. 2003, Rep. Prog. Phys., 66, 2025

Mak, M. K., \& Harko, T. 1999, Classical Quantum Gravity, 16, 4085

Meszaros, P., \& Rees, M. J. 1994, MNRAS, 269, L41

Mitrofanov, I. G. 2003, Nature, 426, 139

Murphy, M. T., Webb, J. K., \& Flambaum, V. V. 2003, MNRAS, 345, 609

Murphy, M. T., et al. 2001, MNRAS, 327, 1208

Overduin, J. M., \& Wesson, P. S. 1997, Phys. Rep., 283, 303

Peebles, P. J. E., \& Ratra, B. 2003, Rev. Mod. Phys., 75, 559

Plaga, R. 1995, Nature, 374, 430

Poirier, J., D’Andrea, C., Fragile, P. C., Gress, J., Mathews, G. J., \& Race, D. 2003, Phys. Rev. D, 67, 042001

Randall, L., \& Sundrum, R. 1999a, Phys. Rev. Lett., 83, 3370 1999b, Phys. Rev. Lett., 83, 4690

Sasaki, M., Shiromizu, T., \& Maeda, K. 2000, Phys. Rev. D, 62, 024008

Shiromizu, T., Maeda, K., \& Sasaki, M. 2000, Phys. Rev. D, 62, 024012

Sommer, M., et al. 1994, ApJ, 422, L63

Stecker, F. W. 2003, Astropart. Phys., 20, 85

Stecker, F. W., de Jager, O. C., \& Salamon, M. H. 1996, ApJ, 473, L75

Uzan, J. P. 2003, Rev. Mod. Phys., 75, 403

Wang, X. Y., Cheng, K. S., Dai, Z. G., \& Lu, T. 2004, ApJ, 604, 306

Webb, J. K., et al. 1999, Phys. Rev. Lett., 82, 884

Weekes, T. C. 2003, in Proc. 28th Int. Cosmic Ray Conf. (Tsukuba, Japan)

Weekes, T. C., et al. 2002, Astropart. Phys., 17, 221

Witten, E. 1996, Nucl. Phys. B, 460, 335

Zhang, B., \& Meszaros, P. 2004, Int. J. Mod. Phys. A, in press (astro-ph/ 0311321) 